\title{
Der digitale Wandel als Motor der Hochschulentwicklung \\ - Strategiemuster für die Digitalisierung der Hochschullehre
}

\section{Jannica Budde}

\section{Zusammenfassung}

Der digitale Wandel in Studium und Lehre stellt Hochschulen vor die Herausforderung, einen Veränderungsprozess strategisch $\mathrm{zu}$ gestalten, dessen Komplexität nicht zu unterschätzen ist. Denn einerseits soll die Digitalisierung kein Selbstzweck sein und in allen strategischen Prozessen mitgedacht werden, andererseits müssen sich Hochschulen auch konkret damit auseinandersetzen, was der digitale Wandel für Studium und Lehre an der jeweiligen Institution bedeutet. Dafür sollte ein individuelles Strategienarrativ entwickelt werden, das den Veränderungsprozess begründet und diesem eine konkrete Richtung gibt. Dieser Beitrag diskutiert die Fragestellung, welche Grundmuster es für diese strategischen Narrative gibt. Dazu betrachtet der Beitrag unterschiedliche Modelle zu Strategie- und Profilierungsoptionen aus der Literatur und entwickelt hieraus ein Modell von sechs Strategiemustern, die als Begründung und Leitrahmen für strategische Digitalisierungsbestrebungen an Hochschulen dienen können.

\section{Schlüsselwörter}

Digitaler Wandel • Digitale Transformation • Strategie • Profilierung • Hochschulentwicklung • Literaturanalyse • Change Management • Strategieoptionen • Peer-to-Peer-Strategieberatung • Innovationsmanagement

\footnotetext{
J. Budde $(\varangle)$

CHE Centrum für Hochschulentwicklung, Hochschulforum Digitalisierung, Gütersloh, Deutschland

E-Mail: Jannica.Budde@CHE.de
} 
Die Digitalisierung von Studium und Lehre erfordert von Hochschulen einen strategisch gesteuerten Veränderungsprozess. Dieser ist dabei in seiner Komplexität nicht zu unterschätzen. Denn einerseits soll die Digitalisierung kein Selbstzweck sein und in allen strategischen Prozessen mitgedacht werden, andererseits müssen sich Hochschulen auch in einem spezifischen Prozess damit auseinandersetzen, welche Bedeutung der digitale Wandel für die jeweilige Institution hat. Gleichzeitig zeichnet sich innerhalb der deutschen Hochschullandschaft, etwa durch die wachsende Diversifizierung der Studierenden, die Notwendigkeit einer stärkeren Profilbildung von Hochschulen ab (Ziegele et al. 2019). Der digitale Wandel kann damit als ein Motor der Hochschulentwicklung dienen. Doch was bedeutet dies genau für die Strategieentwicklung im Bereich Studium und Lehre, welche inhaltlichen Handlungsräume haben Hochschulen bei der Entwicklung eines strategischen Leitbildes für die Hochschulbildung im digitalen Zeitalter?

\section{Veränderungsprozesse in Studium und Lehre gestalten: Change Management versus Diffusionsprozesse}

Es ist ein schon oft wiederholter Allgemeinplatz, dass der digitale Wandel in Studium und Lehre - sei es nun die Einführung digitaler Technologien oder die curriculare Verankerung von sogenannten Digital- und Zukunftskompetenzen - einen strategisch gesteuerten Veränderungsprozess vonseiten des Hochschulmanagements benötigt. Ziel ist hierbei die nachhaltige Implementation von Innovationen und Strukturen im Sinne einer Restabilisierung des Systems, wie es Kurt Lewin 1958 in seinem Dreiphasenmodell beschreibt. Denn während die spezifische Kultur an deutschen Hochschulen durch ihre Dezentralität Innovationen in Lehr-/Lernmethoden, -technologien und -szenarien fördert, stellt diese auch ein Hemmnis für die hochschulweite nachhaltige Verankerung von Innovationen und damit den digitalen Wandel im Ganzen dar.

Innovationen und Veränderungen in der Lehre sind abhängig vom individuellen Commitment von Lehrenden. Damit ist die digitale Transformation an Hochschulen mit Rogers (2003) zunächst als Diffusionsprozess zu verstehen. Die Übernahme von Innovationen ist abhängig von individuellen Einstellungen bezüglich der digitalen Transformation und der individuellen Einstellung gegenüber Neuerungen. Diffusionsprozesse sind also auch abhängig vom Wissen über die jeweilige Innovation, etwa zu ihrem Nutzen. Damit sich technische und soziale Innovationen im Kontext der Digitalisierung von Studium und Lehre durchsetzen, 
braucht es also auch immer formelle wie informelle Wege der Wissensvermittlung. Die bloße Verteilung von Methoden, Werkzeugen und Lehr-/Lernszenarien ist nicht ausreichend für eine nachhaltige Implementation von Innovationen.

Da der Diffusionsprozess abhängig ist vom Erfolg der einzelnen Innovation (technischer oder sozialer Natur), lässt sich behaupten, dass sich der digitale Wandel nur bedingt (strategisch) steuern lässt. Eine strategische Ausrichtung erleichtert und unterstützt jedoch diesen Wandlungsprozess und kann den Diffusionsprozess beschleunigen. ${ }^{1}$ Daher ist es notwendig, dass Hochschulleitungen passende Strukturen für eine nachhaltige Verankerung entwickeln, etwa in Form von Curriculumentwicklung, entsprechenden Infrastrukturen und Supportstrukturen sowie strategischer Personalentwicklung. Weiterhin gehören dazu Strukturen, die dabei helfen, Leuchtturmprojekte strategisch auszurichten und bei Erfolg in vorhandenen Strukturen zu verankern. Budde und Oevel (2016) sprechen hier von einem Innovationsmanagement für Hochschulen. Ein Refreezing im Sinne Lewins (1958) wäre dann als Implementation von Innovationen, beispielsweise Softwareprodukten, in zentralen Strukturen (zum Beispiel Bibliothek, Medienzentrum, IT) oder Fakultätsstrukturen zu verstehen. Dies ist notwendig, da einzelne Lehrende in der Regel nicht in der Lage sind, ein in einem Projekt entwickeltes Tool als ein dauerhaftes Produkt zu betreiben. Dieses Beispiel macht deutlich, wie wichtig es ist, dass passende Strukturen für die nachhaltige Implementation von Lehrinnovationen durch strategische Entscheidungs- und Entwicklungsprozesse aufgebaut und weiterentwickelt werden.

\section{Strategiemuster für die digitale Transformation}

Der digitale Wandel konfrontiert Hochschulen in Bezug auf ihr Kerngeschäft Studium und Lehre mit einer recht komplexen Situation, eine Lehrstrategie für das digitale Zeitalter ist keine „Strategie wie jede andere“ (Schünemann und Budde 2018). Hinzu kommt, dass es für Lehrstrategien im digitalen Zeitalter als Teil der individuellen Profilentwicklung einer jeden Hochschule keine Einheitslösungen

\footnotetext{
${ }^{1}$ Wichtig ist, dass es dabei darum geht, einzelne Aspekte der Digitalisierung profilunterstützend hervorzuheben und deren Verbreitung durch gezielte Maßnahmen im Sinne eines Innovationsmanagements zu untermauern, und nicht darum, alle Lehrenden auf eine Linie zu bringen. Vielmehr braucht es neben einem zielgerichteten Narrativ auch eine Ermöglichungskultur, um die Lehre durch neue Technologien weiterzuentwickeln und Lehrenden die verbriefte Freiheit der Lehre zu gewähren. Hierbei gilt es dann, einen dynamischen Strategieprozess in Gang zu setzen, der kontinuierlich neue Entwicklungen berücksichtigt.
} 
geben kann. Vielmehr muss jede Hochschule für sich klären, was eine Strategie für das digitale Zeitalter inhaltlich für die eigene Institution bedeutet. Hierbei gilt es 1) im Prozess auszuhandeln und zu definieren, was genau in der aktuellen Diskussion vor Ort unter Digitalisierung und digital unterstütztem Lehren und Lernen zu verstehen ist, und 2) das Warum zu klären, also ein Narrativ ${ }^{2}$ oder einen Leitrahmen für die Digitalisierung passend zum übergreifenden Leitbild der eigenen Hochschule zu entwickeln. Aus diesem entwickelten Narrativ wiederum sollte idealerweise 3) ein möglichst konkreter Handlungsrahmen im partizipativen Prozess entwickelt werden, der ausreichende Handlungsfreiräume auf den unterschiedlichen Fach- und Entscheidungsebenen ermöglicht (Schmid und Baeßler 2016, S. 48). Ein derart beschriebenes Vorgehen erleichtert den eigentlichen Strategieprozess im Sinne der Entwicklung von strategischen Zielen und die Identifikation passgenauer Maßnahmen, die ressourcenschonend entsprechend den Zielen priorisiert werden können. Darüber hinaus können ein solches Narrativ und ein partizipatives Vorgehen die Akzeptanz für den Veränderungsprozess aufseiten der Fakultäten/Fachbereiche und einzelnen Lehrenden erhöhen.

Doch welche thematischen Handlungsräume haben Hochschulen bei der Entwicklung eines strategischen Leitbildes für die Hochschulbildung im digitalen Zeitalter? Gibt es trotz individueller Eigenheiten spezifische Grundmuster für die strategischen Narrative? Dazu werden im Folgenden unterschiedliche Modelle von Strategieoptionen untersucht, um anschließend ein eigenes Modell zu entwickeln.

Die Entwicklung eines Narrativs oder Leitrahmens für die Digitalisierung hat viele Vorteile für den Strategieprozess. Dies ermöglicht eine klare Priorisierung, führt aber auch zur Aushandlung und Entscheidung, was genau mit Digitalisierung gemeint ist und wo genau Veränderungen im Sinne einer digitalen Transformation ansetzen sollen: Die Komplexität des Strategiethemas Digitalisierung der Hochschullehre/-bildung zeigt sich daher zunächst einmal in den unterschiedlichen Möglichkeiten der Herangehensweise, angefangen bei der Modernisierung der Hochschulstrukturen im Sinne eines Ausbaus der Infrastruktur und der Digitalisierung von administrativen Unterstützungsprozessen über die strategische Einführung von digitalen Lehr-/Lerntechnologien und digital unterstützten Lehr-/ Lernszenarien (digital unterstütztes Lehren und Lernen) bis hin zur Entwicklung einer Strategie für das digitale Zeitalter, die weniger die Lehrmethoden, sondern vielmehr Lerninhalte und Kompetenzentwicklung für ein digitales Zeitalter in den Mittelpunkt rückt. Des Weiteren und parallel dazu kann die Digitalisierung auch

\footnotetext{
${ }^{2}$ Mit von Stuckrad und Röwert lässt sich ein Narrativ oder eine Story als eine in eine Erzählung eingebettete Erklärung des strategischen Profils beschreiben oder als ,alltagssprachlich und alltagspraktisch anschlussfähiger, roter Faden““ (von Stuckrad und Röwert 2017, S. 2).
} 
als Mittel zum Zweck, das heißt als Ausgangspunkt und zur Unterstützung anderer strategischer Ziele dienen. Hochschulleitungen und weitere Akteur*innen der Strategieentwicklung haben damit für den Bereich Lehre eine große Auswahl an Möglichkeiten, wo sie im Strategieprozess ansetzen können. ${ }^{3}$ Mit den genannten Aspekten eröffnet sich eine Spannbreite in Bezug auf die Digitalisierung, die von der (technologischen) Modernisierung der Hochschullehre bis hin zur Profilbildung durch Digitalisierung reicht.

\subsection{Modernisierung versus Profilierung}

Getto und Kerres (2017) unterscheiden in Bezug auf die Digitalisierung zwischen Modernisierung und Profilierung als den zwei Handlungsoptionen für Hochschulen. Dies hänge zunächst einmal mit dem (meist) impliziten Verständnis von Digitalisierung als „Modernisierungstrend“ oder als „Chance zur Profilierung“ (S. 123) zusammen. Sie verbinden diese beiden Optionen mit unterschiedlichen Verständnissen von Hochschulen: „Ein institutionelles Verständnis von Hochschule würde die Digitalisierung eher als einen allgemeinen Modernisierungstrend auffassen, der weitgehend gleichförmig einzuführen ist. Ein organisationales Verständnis von Hochschule würde die Digitalisierung dagegen eher als Chance zur Profilierung einer Hochschule im Wettbewerb auffassen“" (S. 127). Reine Modernisierung ist für Getto und Kerres jedoch keine genuin strategische Aufgabe. Hechler und Pasternack (2017) entwickeln ein ähnliches binäres Schema und unterscheiden zwischen der Digitalisierung als Zusatz zu traditionellen Formaten und Prozessen auf der einen und Digitalisierung als transformativen Prozess von Lehren und Lernen auf der anderen Seite. Modernisierung bezieht sich in diesem Sinne eher auf die technologische Ebene, kann aber selbst wieder in administrative Prozesse der Lehrorganisation (zum Beispiel Campusmanagementsysteme) sowie die didaktisch-methodische Dimension der Lehre unterschieden werden (zum Beispiel Einführung von Flipped Classrooms oder E-Portfolios). Dies entspricht auch der Behauptung von Dräger et al. (2017), Modernisierung beschränke sich ,auf eine systematische Koordination und Organisation der Problemlösungspotentiale der Digitalisierung“ (S. 267). Es gehe beim Modernisierungsansatz eher darum, digitale Lösungsstrategien für existierende Herausforderungen zu finden, wozu auch der Ausbau der Infrastruktur zähle. Eine „IT-gestützte Modernisierung

\footnotetext{
${ }^{3}$ Hinzu kommt die Notwendigkeit, die Strategieentwicklung für den Bereich Lehre samt den daraus abgeleiteten Maßnahmen in eine Digitalisierungsstrategie einzubetten, die alle Bereiche der Hochschule (also auch Forschung, Verwaltung und Third Mission) abdeckt und Maßnahmen in diesen Bereichen aufeinander abstimmt.
} 
der Lehre [ist] für sich genommen noch kein starkes profilbildendes Element", behaupten auch Schmid und Baeßler (2016, S. 9). Als ein Handlungsfeld für die Modernisierung der Lehre verweisen Dräger et al. (2017) auf die Herausforderung der wachsenden Studierendenschaft sowie die wachsende Diversität der Studierenden. Im Gegensatz zu einer profilbildenden Maßnahme ist in ihrem Verständnis ein Modernisierungsprozess also viel stärker von gesellschaftlichen Veränderungen evoziert, Hochschulen fühlen sich hier also mehr als Getriebene der Digitalisierung denn als deren aktive Gestalter.

\subsection{Profilbildungs- und Profilierungsoptionen}

Diese Auseinandersetzung mit den beiden Digitalisierungsparadigmen lässt vermuten, dass zwischen Modernisierung und Profilierung nicht unbedingt aufgrund unterschiedlicher inhaltlicher Themen unterschieden werden kann, sondern durch die Herangehensweise an das Thema Digitalisierung der Lehre. Bereits 2007 konstatieren Euler und Seufert „Reaktivität“ und „Proaktivität“ als Unterscheidungskriterium zwischen den unterschiedlichen „Innovationsfoki“: „Dabei ist wesentlich, ob eine Optimierung als Reaktion auf Umfeldveränderungen oder eher ein strategischer Wandel und das proaktive Ergreifen neuer Chancen angestrebt werden soll“" (S. 10). Sie stellen dabei den Innovationsfokus der Strategie in Beziehung zu der "Innovationsausrichtung“ und entwickeln die folgende Typologie der strategischen Ausprägungsformen von E-Learning-Strategien:

1. Professionalisierungsstrategie

2. Flexibilisierungsstrategie

3. Reformstrategie

4. Vermarktungsstrategie

Professionalisierungs- und Flexibilisierungsstrategie werden als „Optimierungsstrategien" bezeichnet und können daher eher dem Modernisierungsparadigma zugeordnet werden. Mit Euler und Seufert (2007) hätte die Modernisierung demnach auch strategische Relevanz oder kann als Strategiethema klassifiziert werden. Der Schwerpunkt der Implementierung dieser Strategie läge damit auf den Organisationsstrukturen und -abläufen. Die Reformstrategie lässt sich dem Profilierungsparadigma zuordnen, da sie auf radikale Veränderungen in der Lehre abzielt, die der Hochschule einen Wettbewerbsvorteil verschaffen sollen. Die Vermarktungsstrategie lässt sich dagegen eher als radikale Profilierungsstrategie verstehen, da sie nach neuen Geschäftsmodellen für den Hochschulsektor sucht. 
Dementsprechend lässt sich mit Schmid und Baeßler (2016) folgern: „Erst wenn die [durch digitale Technologien] ermöglichten Potentiale dazu genutzt werden, einer Hochschule strategische Wettbewerbsvorteile oder andere Alleinstellungsmerkmale zu verschaffen“ (S. 9), könne man von einer Profilbildung im eigentlichen Sinne sprechen. Für eine Reformstrategie im Sinne Eulers und Seuferts (2007) bedarf es also auch immer etwas, das zum Alleinstellungsmerkmal gemacht werden kann, etwas, das oben Narrativ und Leitrahmen zur Digitalisierung der Hochschullehre genannt worden ist. Hier definiert die Hochschule konkret, wozu die Möglichkeiten der Digitalisierung eingesetzt werden sollen und fokussiert sich zugleich auf eine oder wenige Optionen. Diese „Handlungsfelder der Digitalisierung" mögen für die jeweilige Hochschule höchst individuell ausformuliert werden, lassen sich aber zu bestimmten Strategieoptionen zusammenfassen. Als „Profilbildungsoptionen“ schlagen Schmid und Baeßler (2016) auf Basis einer Literaturrecherche die folgenden fünf Kategorien ${ }^{4}$ vor:

1. Internationalisierung

2. Individualisierung

3. Anwendungsorientierte Lehre

4. Forschungsorientierte Lehre

5. Weiterbildung

Die Autor*innen machen anhand von Praxisbeispielen jedoch auch sehr gut deutlich, wie die Profilbildungsoptionen ineinandergreifen, etwa am Beispiel der TU Dresden:

„Erstes Ziel ist die Verbesserung der Studierbarkeit und Erhöhung des Studienerfolgs. Vor dem Hintergrund steigender Studierendenzahlen und der starken Heterogenität der Studierendengruppen sollen digitale Lernsettings dabei helfen, individuelle und interaktive Lernprozesse auch im Massenbetrieb umzusetzen, z. B. durch onlinegestützte Assessments und Prüfungen. [...] Schließlich öffnet sich durch die zeitlich und örtlich flexible digitale Lehre die Universität auch neuen Zielgruppen und erweitert die Lehre in Richtung Lebenslanges Lernen“" (S. 34 f.).

\footnotetext{
${ }^{4}$ Diese Kategorien wurden anhand von ausgewählten deutschen Hochschulen validiert. Dabei zeigte sich, dass keine der Hochschulen nur eine einzige Ausrichtung verfolgte, sondern dass jeweils mindestens zwei Profilbildungspotenziale von den Forscher*innen gefunden werden konnte. Dennoch lässt sich auch jeweils ein deutlicher Schwerpunkt auf eine Kategorie identifizieren.
} 
Dräger et al. (2017) dagegen sehen die Chance der Digitalisierung vor allem in der Möglichkeit der Profilbildung und der Schärfung der institutionellen Identität. Sie identifizieren daher die folgenden „Profilierungsoptionen“:

1. Die diversitätsgerechte Hochschule

2. Die berufsbegleitende Hochschule

3. Die Hochschule mit offenem Zugang

4. Die Guidance-Hochschule

5. Die im Verbund agierende Hochschule

6. Die gesellschaftsorientierte Hochschule

7. Die Anerkennungshochschule

8. Die auf Produktion digitaler Lehre spezialisierte Hochschule ${ }^{5}$

Die hier formulierten Optionen verweisen weniger auf Potenziale der Digitalisierung für das individuelle Lehren und Lernen, sondern vielmehr auf die strategische Ausrichtung der Hochschule als Ganzes. Daneben werden auch thematische Profiloptionen sichtbar, wie Diversität/Individualisierung und Weiterbildung sowie in Ergänzung zu bisherigen Modellen die Öffnung der Hochschule. ${ }^{6}$

\subsection{Handlungsoptionen von Hochschulen}

Zur Vertiefung der Fragestellung, was Hochschulen unter dem Stichwort Digitalisierung der Hochschullehre strategisch bezwecken möchten, wurden Interessenbekundungen zur Peer-to-Peer-Strategieberatung des Hochschulforums Digitalisierung (HFD) stichprobenartig ausgewertet. Die wichtigsten Ergebnisse und Thesen wurden in einem Diskussionspapier veröffentlicht (Watolla 2019).

\footnotetext{
${ }^{5}$ Gerade die Optionen 7 und 8 lassen sich mit Euler und Seufert (2007) als Vermarktungsstrategien oder in unserer Terminologie als radikale Profilierungsstrategien beschreiben. Da beide Optionen noch relativ selten sind, sowohl im deutschen als auch im internationalen Kontext, sollen radikale Profilierungsstrategien in diesem Beitrag nicht weiterverfolgt werden.

${ }^{6}$ Die meisten Beispiele beschreiben in unterschiedlichen Spielarten die Themen Flexibilisierung des Studiums und den Umgang mit nicht-traditionellen Studierenden - Aspekte, die Dräger et al. (2017) zunächst auch im Kontext der Modernisierung diskutieren. Dies entspricht auch der Typologisierung nach Euler und Seufert (2007), die in Reaktion auf die veränderte Zusammensetzung der Studierendenschaft vor allem eine organisatorische Herausforderung sehen und diese daher den Optimierungs- und Modernisierungsstrategien zurechnen (siehe oben).
} 
Die Peer-to-Peer-Strategieberatung ist ein Angebot des Hochschulforums Digitalisierung und stellt ein strategisches Instrument zur Unterstützung von Hochschulleitungen bei der Strategieentwicklung dar (Hochschulforum Digitalisierung 2020). Die Interessenbekundung ist eine formlose Bewerbung zu diesem Programm, wobei jeweils sechs Hochschulen auf dieser Basis ausgewählt werden. In den Interessenbekundungen sollen die Hochschulen mindestens zwei Handlungsfelder angeben. Unter strategischen Handlungsfeldern werden die von der Hochschule gesetzten inhaltlichen Schwerpunkte in der Entwicklung einer Strategie für das digitale Zeitalter verstanden. Entlang dieser Handlungsfelder benennen Hochschulen konkrete Ziele, die sie mit der Digitalisierung in der Hochschullehre verfolgen. Folgende zentrale Handlungsfelder wurden im deduktiven und induktiven Verfahren identifiziert und systematisiert (Watolla 2019):

1. Vorbereitung auf die digitale Arbeitswelt

2. Verbesserung der Lehre

3. Flexibilisierung der Lehre

4. Individualisierung in der Lehre

5. Erhöhung des Studienerfolgs

6. Curriculumentwicklung

7. Internationalisierung

Abgesehen von Internationalisierung lassen sich die von Watolla identifizierten Handlungsfelder als Ausdifferenzierung des strategischen Ziels „Weiterentwicklung der Lehre für ein digitales Zeitalter" verstehen, das in der Ausschreibung zur Peer-to-Peer-Beratung benannt wird (Hochschulforum Digitalisierung 2019). Wie Abb. 1 zeigt, haben die meisten Hochschulen, die sich für das Beratungsprogramm bewerben, schon recht konkrete Vorstellungen, wo die digitale Transformation der grundständigen Lehre ansetzen kann. Jedoch zeigt die Tatsache, dass die Zielvorstellung Verbesserung der Lehre immer noch in der Stichprobe auftaucht, dass es weiterhin Hochschulen gibt, die noch keine klaren Mehrwerte für sich benennen konnten. Im Strategieprozess sollten sich die Beteiligten und insbesondere die Hochschulleitungen jedoch immer bewusst machen, warum sie eine Verbesserung der Lehre mithilfe der Digitalisierung anstreben und was sie darunter verstehen. Ziel muss es sein, ein sinnstiftendes Narrativ zu entwickeln, hinter dem sich die Angehörigen der jeweiligen Hochschule versammeln können und das handlungsweisend eine Orientierung für die Operationalisierung der Strategie bietet.

Das wichtigste Narrativ scheint für die Hochschulen die Vorbereitung der Studierenden auf die digitale Arbeitswelt zu sein, wobei die Kompetenzorientierung 


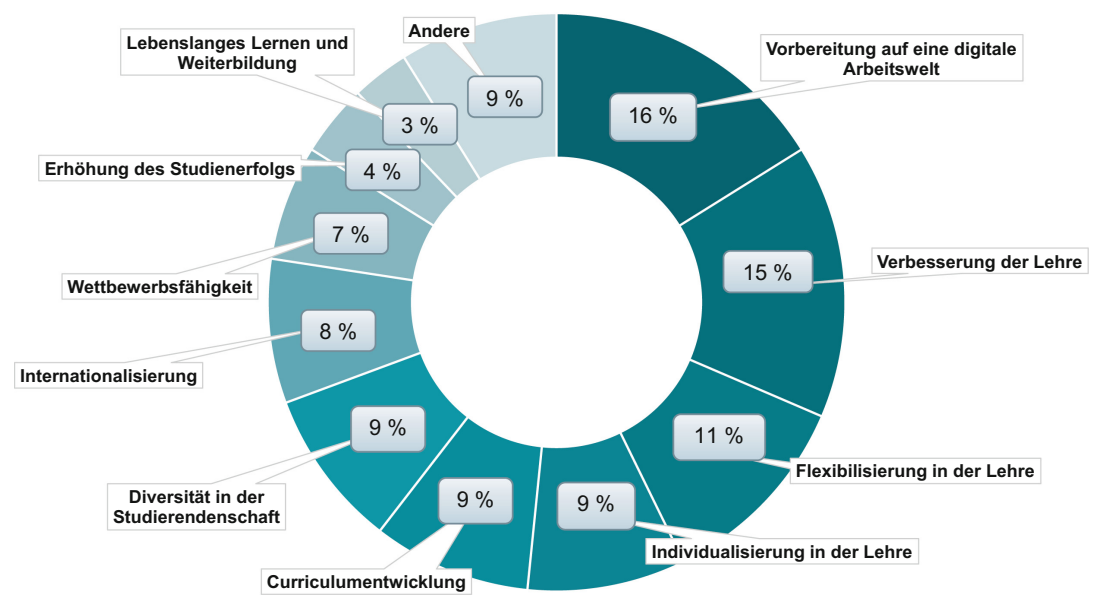

Abb. 1: Herausforderungen in den Interessenbekundungen der Peer-to-Peer-Beratung. (Budde nach Watolla)

naturgemäß sehr stark bei den Fachhochschulen und Hochschulen für angewandte Wissenschaften vertreten sein dürfte. Allerdings zeigte die stichprobenartige Analyse der Interessenbekundungen auch, dass es in diesem Handlungsfeld noch weiterer Ausdifferenzierungen bedarf, um die Kompetenzanforderungen für Studierende konkreter zu beschreiben. Nur so kann das von einer Hochschule entwickelte Narrativ der Kompetenzorientierung in strategischen Prozessen umgesetzt werden. ${ }^{7}$ Das Handlungsfeld Curriculumentwicklung ist dagegen weniger eine inhaltliche Zieldefinition an sich als ein Handlungsfeld im Strategieprozess ${ }^{8}$ und gehört eng zum Themenfeld Kompetenzentwicklung/Vorbereitung auf die digitale Arbeits- und Lebenswelt. Die Handlungsfelder Flexibilisierung und Individualisierung entsprechen den von Schmid und Baeßler (2016) vorgeschlagenen „Strategieoptionen“. Mit diesen beiden Handlungsfeldern beschreiben die Hochschulen vielmehr, wie Lehre im digitalen Zeitalter aussehen kann. Warum Lehre flexibilisiert und individualisiert werden soll, bleibt also erst einmal unklar. Beide Optionen können jedoch dem Handlungsfeld „Erhöhung des Studienerfolgs“ zugeordnet werden sowie als Maßnahme zur Adressierung einer heterogenen Studierendenschaft gelten.

\footnotetext{
${ }^{7}$ Ich danke Ann-Kathrin Watolla für diesen Hinweis.

${ }^{8}$ Siehe: https://hochschulforumdigitalisierung.de/de/dimensionen-des-digitalen-wandels. Zugegriffen: 29.10.2020.
} 


\subsection{Strategiemuster für den Digitalisierungsprozess an Hochschulen in den Bereichen Studium und Lehre}

Auf Basis der Literaturanalyse sowie der Auswertung der Interessenbekundungen zur Peer-to-Peer-Strategieberatung lassen sich folgende Grundmuster für ein strategisches Digitalisierungsnarrativ oder Begründungsmuster für die digitale Transformation in Studium und Lehre identifizieren:
A. Vorbereitung auf die digitale Arbeitswelt
B. Adressierung von Diversität der Studierenden
C. Erhöhung des Studienerfolgs
D. Internationalisierung
E. Öffnung der Hochschule
F. Lebenslanges Lernen und Weiterbildung 9

Im Gegensatz zu den Strategieoptionen von Schmid und Baeßler (2016) sollen mit diesen Mustern weniger die Möglichkeiten der strategischen digitalen Transformation von Lehre in den Blick genommen werden als die potenziellen Ziele, die mit Hilfe von Digitalisierung (basierend auf dem übergreifenden Leitbild der Hochschule) erreicht werden sollen - quasi als gesamtinstitutionelle Begründung, warum eine Hochschule sich mit dem Thema Digitalisierung von Studium und Lehre beschäftigen will und wozu digital unterstützte Lehr-/Lern- und Prüfungsformate sowie digitale Tools zum Einsatz kommen sollen. ${ }^{10}$ Allerdings bedeutet dies auch, dass das Narrativ mit bestimmten Strategieoptionen und passenden Lösungsansätzen verbunden werden muss. Dies wird in den von Schmid und Baeßler (2016) untersuchten Praxisbeispielen sehr anschaulich. So verbindet die TU Dresden, wie oben bereits aufgeführt, mit der Digitalisierung das Ziel, die

\footnotetext{
${ }^{9}$ Die Zieloptionen A bis C befassen sich mit der grundständigen Lehre im Zentrum der Hochschullehre. D bis G lassen sich dagegen eher der Peripherie zuordnen. Die peripheren Bereiche bieten Potenzial, um die Dissemination von digitalen Lehrformaten sowie Lehrformaten, die digitale Kompetenzen vermitteln, zu unterstützen, da hier weniger Widerstände zu erwarten sind. Damit können diese Bereiche den Veränderungsprozess an Hochschulen anstoßen, was wiederum zu Best-Practice-Erfahrungen führt.

${ }^{10}$ Alle diese Themen sind relevant für Hochschulen und ihren Digitalisierungsprozess. Für einen iterativen Strategieprozess ist es jedoch wichtig, sich auf einzelne Aspekte zu konzentrieren, Aspekte zu priorisieren und andere nachzulagern (Schünemann und Budde 2018). Ziel des Klärungsprozesses sollte es sein, dass bestimmte Aspekte für die Profil- und Weiterentwicklung und Unterstützung des Hochschulprofils herausgegriffen werden und in Form eines Narrativs im Fokus der Strategieentwicklung stehen, um dadurch wesentlich mehr Stakeholder auf den verschiedenen Ebenen im Prozess mitzunehmen.
} 
Studierbarkeit ihrer Studiengänge zu verbessern und den Studienerfolg zu erhöhen, indem sie auf die Strategieoption Individualisierung der Lehre setzt. Ebenso kann die von Schmid und Baeßler (2016) genannte Praxis- und Forschungsorientierung eine Strategieoption für das Ziel sein, die Studierenden bestmöglich für die Zukunft auszubilden.

\section{Digitalisierungsnarrative im Kontext des Change Management}

Die Digitalisierungsnarrative, die in den aus der Literatur herausgearbeiteten Strategiemustern enthalten sind, stellen wichtige Elemente für einen strategisch unterstützten Digitalisierungsprozess von Studium und Lehre dar. Die Aushandlung und Formulierung eines Digitalisierungsverständnisses samt einem an den Hochschulalltag anknüpfenden Leitrahmen in Form einer ,in eine Erzählung eingebetteten Erklärung“ (Von Stuckrad und Röwert 2017, S. 2), wozu die digitale Transformation in Studium und Lehre zum Einsatz kommen soll, ermöglicht die Entwicklung und Priorisierung von konkreten strategischen Maßnahmen. Hierzu ist es notwendig, dem jeweiligen Narrativ die richtigen Strategieoptionen zuzuordnen und mit Entscheidungen für Infra- und Unterstützungsstrukturen zu unterlegen. ${ }^{11}$ Für die an den Hochschulen gewachsenen Strukturen bedeutet dies auch, bestehende Angebote darauf zu prüfen, ob sie zu den mit dem Narrativ formulierten strategischen Zielen beitragen, und dann ggf. auch (vorerst) einzustellen. Durch ein Narrativ kann ein Veränderungsprozess in Studium und Lehre also dadurch eingeleitet werden, dass das Verhalten von Lehrenden einerseits durch eine klare Richtungsanweisung sowie andererseits durch passende Infrastrukturen und Unterstützungsangebote, also entsprechende strategisch gewollte Möglichkeitsräume, beeinflusst wird. ${ }^{12}$ Hierzu gehört auch ein Innovationsmanagement, das diese strategische Ausrichtung beispielsweise durch

\footnotetext{
${ }^{11}$ Eine Zuordnung von Strategieoptionen und konkreten Maßnahmen zu den herausgearbeiteten Strategieoptionen kann in diesem Beitrag nicht vorgenommen werden.

${ }^{12}$ Hierbei ist zu beachten, dass strategische Leitrahmen und Narrative der Digitalisierung inhärenten Beschleunigung und Weiterentwicklung nicht im Wege stehen sollen und damit die Lehre gewissermaßen auf dem aktuellen Stand konservieren. Vielmehr müssen Digitalisierungsnarrative stetig auf ihre Wirksamkeit und ihre enthaltenen Strategieoptionen hin überprüft, weiterentwickelt und wenn nötig revidiert werden. Hierzu braucht es die Innovationsfähigkeit der Lehrenden und Studierenden durch strategisch ermöglichte Frei- und Experimentierräume.
} 
Projektförderungen und Einbindung strategischer Projekte in zentrale Strukturen in den Blick nimmt.

Im Sinne von Rogers Diffusionsmodell ist es dabei wichtig, Widerstände von Lehrenden (und Studierenden!) bezüglich der digitalen Transformation im Allgemeinen und des jeweiligen strategischen Narrativs im Besonderen zu minimieren. Dazu ist nicht nur ein gutes Kommunikationsmanagement vonseiten der Hochschule sowie die Einbindung aller Akteursgruppen in den Strategieprozess wichtig. Es bedarf auch einer schlüssigen Herleitung des Digitalisierungsnarrativs aus der Alltagspraxis von Studium und Lehre sowie einer Synchronisation mit dem Selbstbild der Hochschule.

Die Synchronisation von strategischen Zielen im Kontext der Digitalisierung der Hochschullehre und den übergeordneten strategischen Zielen ist also einer der wichtigsten Erfolgsfaktoren für die Verbreitung und Verankerung von Digitalisierung in der Hochschullehre:

\begin{abstract}
„Adäquate Strategien für das digitale Zeitalter verknüpfen die institutionelle Identität unmittelbar mit digitalen Angeboten. [...] Hochschulen können und sollten die Chancen der Digitalisierung nutzen, um das gewünschte Profil, das angestrebte Angebotsportfolio, die intendierten Zielgruppen und die definierten strategischen Entwicklungsziele besser zu erreichen“ (Dräger et al. 2017, S. 268 f.).
\end{abstract}

Die Digitalisierung kann damit wesentlich zur Umsetzung des institutionellen Profils beitragen; teilweise wäre diese ohne Digitalisierung gar nicht realisierbar. Die Fallbeispiele, die Schmid und Baeßler (2016) in ihrer Studie untersuchen, zeigen alle eine hohe Synchronisation der Ziele mit den übergeordneten Leitbildern auf, sodass davon auszugehen ist, dass Hochschulen weniger Schwierigkeiten haben, ihre strategischen Ziele an das Leitbild zu koppeln, sobald sie sich von der Auffassung von Digitalisierung als Selbstzweck gelöst haben. Dies sollte jedoch in einer weiteren Studie auf Basis der identifizierten Strategiemuster validiert werden.

\title{
$4 \quad$ Fazit und Ausblick: Hochschule, quo vadis?
}

Hochschulleitungen und strategische Entscheider*innen an Hochschulen wissen um die Bedeutung des transformativen Potenzials der Digitalisierung in Richtung des mit dem Bologna-Prozess angestoßenen Shift from Teaching to Learning. Die Digitalisierung ermöglicht hierbei, Lehren und Lernen neu zu gestalten. Aus strategischer Perspektive ist es jedoch ratsam, sich in Bezug auf die Weiterentwicklung der Lehre nicht auf die Digitalisierung per se zu konzentrieren, 
sondern auf Ziele, die im Einklang mit dem Leitbild der Hochschule stehen. Dies bedeutet, dass jede Hochschule, idealerweise in einem alle Stakeholdergruppen umfassenden Verständigungsprozess, für sich selbst strategische Ziele und die dazugehörigen Strategieoptionen - also das Warum und das Wie - entwickeln muss.

In diesem Beitrag wurden anhand einer Literaturanalyse sechs Grundmuster für Strategienarrative in Bezug auf Lehre und Studium im digitalen Zeitalter entwickelt. Im nächsten Schritt müssen diese anhand realer Strategieprozesse, zum Beispiel aus der Peer-to-Peer-Strategieberatung des Hochschulforums Digitalisierung und öffentlich zugänglicher Strategiepapiere, validiert werden. Hieran schließen sich weitere Fragestellungen an, beispielsweise ob sich die Strategiemuster bestimmten Hochschultypen zuordnen lassen, wie sie zu anderen Strategietypen passen und wie sich die Dynamik der Digitalisierung in diesen Strategiemustern wiederfindet. Außerdem sollten aufgrund realer Strategieprozesse Strategieoptionen und Maßnahmen für die jeweiligen Muster abgeleitet werden, um so Hochschulen bei der Umsetzung des individuellen Strategieprozesses zu unterstützen.

Strategienarrative als Leitbilder für die digitale Transformation unterstützen die strategische Implementation der digitalen Transformation in Studium und Lehre, indem sie dabei helfen, Strukturen und Angebote strategisch auszurichten sowie die Dissemination von technischen wie sozialen Innovationen in Bezug auf die Lehre innerhalb der einzelnen Hochschule erleichtern. Akzeptanz entsteht, wenn der Sinn des Change-Prozesses deutlich wird. Ein solches Strategienarrativ bildet auch die Basis eines Innovationsmanagements an Hochschulen, etwa bei der Auswahl, welche internen Projekte gefördert werden sollen. Damit aus dem Narrativ konkrete Unterstützungsmaßnahmen abgeleitet werden können und so einen brauchbaren Rahmen für den Entwicklungsprozess bieten, ist es jedoch notwendig, dass dieses Narrativ an die konkrete Situation der Hochschule angepasst und anhand einer klaren Zielsetzung formuliert worden ist.

Es wurde des Weiteren die Rolle der Unterscheidung zwischen Modernisierung und Profilierung als Paradigmen für Strategieprozesse an Hochschulen diskutiert. Entscheidend ist vor allem die Herangehensweise der jeweiligen Hochschule an den Strategieprozess. Offen bleibt, wie relevant die Unterscheidung für Hochschulen im hier beschriebenen Transformationsprozess ist. Es kann eher davon ausgegangen werden, dass deutsche Hochschulen vorrangig an einer Profilierung durch Modernisierung und Weiterentwicklung des bestehenden Verständnisses von Lehre interessiert sind, das heißt, dass die Digitalisierung das bestehende Lehrprofil der jeweiligen Hochschule unterstützen oder erweitern soll. Die strategisch avisierten Veränderungen in diesen Strategiemustern sind daher eher 
inkrementell als radikal. Dies ist sicherlich auf die große Herausforderung zurückzuführen, dass Hochschulleitungen nicht einfach eine neue Lehre und Lehrinhalte vorschreiben, sondern lediglich Rahmenbedingungen entwickeln können. Radikale Strategieoptionen, die Formen von Hochschulbildung neu denken und neue Geschäftsmodelle entwickeln, wurden daher bisher aus den Betrachtungen in diesem Beitrag ausgeklammert. Für die Zukunft kann jedoch mehr und mehr von einer Diversifizierung der immer noch sehr homogenen deutschen Hochschullandschaft ausgegangen werden. Dies verändert nicht nur die Lehre als solche, sondern auch die Aufgaben von Hochschulen und ihr Angebotsspektrum (Orr et al. 2019; Ziegele et al. 2019). Hochschulen müssen in Zukunft also noch stärker strategische Ziele und Alleinstellungsmerkmale ausbilden.

\section{Literatur}

Budde, J., \& Oevel, G. (2016). Innovationsmanagement an Hochschulen: Maßnahmen zur Unterstützung der Digitalisierung von Studium und Lehre. In C. M. Heinrich \& M. Pinzger (Hrsg.), INFORMATIK 2016 (S. 947-959). Bonn: Gesellschaft für Informatik e. V.

Budde, J., Oevel, G., \& Wilde, M. (2018). Lehr-/Lerninnovationen nachhaltig gestalten - Ein Leitfaden. https://doi.org/10.5281/zenodo.1291408.

Dräger, J., Friedrich, J.-D., Mordhorst, L., Müller, U., \& Röwert, R. (2017). Hochschulen brauchen Strategien für das digitale Zeitalter. In Rat für Forschung und Technologieentwicklung. (Hrsg.), Zukunft und Aufgaben der Hochschulen (S. 263-278), Münster: Lit Verlag.

Euler, D., \& Seufert, S. (2007). Change Management in der Hochschullehre: Die nachhaltige Implementierung von e-Learning-Innovationen. Zeitschrift für Hochschulentwicklung, 3, $3-15$.

Getto, B., \& Kerres, M. (2017). Akteurinnen/Akteure der Digitalisierung im Hochschulsystem: Modernisierung oder Profilierung? Zeitschrift für Hochschulentwicklung, 12(1), 123-142.

Hechler, D., \& Pasternack, P. (2017). Digitalisierungsstrategien und Digitalisierungspolicies an Hochschulen. Die Hochschule: Journal für Wissenschaft und Bildung, 26, 84-105.

Hochschulforum Digitalisierung. (2019). Peer-To-Peer-Beratung zu Strategien für Hochschulbildung im digitalen Zeitalter. https://hochschulforumdigitalisierung.de/de/strate gien-hochschulbildung-digitales-zeitalter. Zugegriffen: 26. März 2020.

Hochschulforum Digitalisierung. (2020). Strategien für Hochschulbildung im digitalen Zeitalter. https://hochschulforumdigitalisierung.de/de/strategien-f\%C3\%BCr-hochschul bildung-im-digitalen-zeitalter. Zugegriffen: 26. März 2020.

Lewin, K. (1958). Group decision and social change. In T. Maccoby, M. Newcomb, \& E. L. Hartley (Hrsg.), Readings in social psychology (S. 197-211). New York: Holt, Rinehart and Winston. 
Orr, D., Lübcke, M., Schmidt, P., Ebner, M., Wannemacher, K., Ebner, M., \& Dohmen, D. (2019). AHEAD - Internationales Horizon-Scanning: Trendanalyse zu einer Hochschullandschaft in 2030 - Hauptbericht der AHEAD-Studie (Arbeitspapier, 42). Berlin: Hochschulforum Digitalisierung. https://doi.org/10.5281/zenodo.2677655.

Rogers, E. (2003). Diffusion of Innovations (5. Aufl.). New York: Free Press.

Schünemann, I., \& Budde, J. (2018). Hochschulstrategien für die Lehre im digitalen Zeitalter: Keine Strategie wie jede andere! (Arbeitspapier, 38). Berlin: Hochschulforum Digitalisierung. https://doi.org/10.5281/zenodo.2592258.

Schmid, U., \& Baeßler, B. (2016). Strategieoptionen für Hochschulen im digitalen Zeitalter (Arbeitspapier, 29). Berlin: Hochschulforum Digitalisierung. https://doi.org/10.5281/zen odo.4282185.

Von Stuckrad, T., \& Röwert, R. (2017). Themenfelder als Profilbildungselement an deutschen Hochschulen: Trendanalyse und Themenlandkarte (Arbeitspapier, 202). Gütersloh: CHE Centrum für Hochschulentwicklung.

Watolla, A.-K. (2019). Strategische Weiterentwicklung von Studium und Lehre im digitalen Zeitalter: Handlungsfelder und Herausforderungen. Diskussionspapier, 6. Berlin: Hochschulforum Digitalisierung. https://doi.org/10.5281/zenodo.3484719.

Ziegele, F., Neubert, P., \& Mordhorst, L. (2019). Die Hochschule der Zukunft: Fels in der Brandung? Hochschulsport, 2, 20-22.

Open Access Dieses Kapitel wird unter der Creative Commons Namensnennung 4.0 International Lizenz (http://creativecommons.org/licenses/by/4.0/deed.de) veröffentlicht, welche die Nutzung, Vervielfältigung, Bearbeitung, Verbreitung und Wiedergabe in jeglichem Medium und Format erlaubt, sofern Sie den/die ursprünglichen Autor(en) und die Quelle ordnungsgemäß nennen, einen Link zur Creative Commons Lizenz beifügen und angeben, ob Änderungen vorgenommen wurden.

Die in diesem Kapitel enthaltenen Bilder und sonstiges Drittmaterial unterliegen ebenfalls der genannten Creative Commons Lizenz, sofern sich aus der Abbildungslegende nichts anderes ergibt. Sofern das betreffende Material nicht unter der genannten Creative Commons Lizenz steht und die betreffende Handlung nicht nach gesetzlichen Vorschriften erlaubt ist, ist für die oben aufgeführten Weiterverwendungen des Materials die Einwilligung des jeweiligen Rechteinhabers einzuholen.

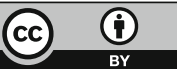

